\title{
Embedded Microchannel Cooling for High Power-Density GaN-on-Si Power Integrated Circuits
}

\author{
Remco van Erp, Georgios Kampitsis, Luca Nela, Reza Soleimanzadeh Ardebili, Elison Matioli \\ Institute of Electrical Engineering \\ École Polytechnique Fédérale de Lausanne (EPFL) \\ Lausanne, Switzerland \\ Email: remco.vanerp@epfl.ch
}

\begin{abstract}
In this work, we demonstrate a new thermal management approach for direct cooling of GaN-on-Si power integrated circuits (ICs), in which the $\mathrm{Si}$ substrate functions as a microfluidic heat sink, turning $\mathrm{Si}$ into a cost-effective, high thermal performance substrate. Flowing coolant through microchannels etched in the backside of the substrate enables a much denser integration of GaN power devices in a single chip. As a proof of concept, an integrated full-wave bridge rectifier (FWBR) was realized based on high-performance tri-anode GaN Schottky barrier diodes (SBDs), together with a novel hybrid printed circuit board (PCB) that provides fluidic and electric connections to the liquid-cooled power IC. A devicelevel heat flux of $417 \mathrm{~W} / \mathrm{cm}^{2}$ was cooled using only $60 \mathrm{~mW}$ of pumping power. Compared to conventional air-cooling, the temperature rise was reduced by $98 \%$ and the converter output power was increased by 30 times, up to $120 \mathrm{~W}$, by eliminating self-heating degradation. The high cooling efficiency, large heat extraction capabilities and low-cost fabrication process of embedded microchannels on GaN-on-Si, in combination with new PCB-based coolant delivery, can be an enabling technology for the next generation of ultra-high power-density ICs.
\end{abstract}

KEY WORDS: microchannels, direct cooling, thermal management, Power IC

\section{NOMENCLATURE}

\begin{tabular}{lll}
\hline Variable & Description & unit \\
\hline COP & Coefficient of performance & - \\
$f$ & Flow rate & $\mathrm{ml} / \mathrm{s}$ \\
$h$ & Heat transfer coefficient & $\mathrm{W} / \mathrm{m}^{2} \mathrm{~K}$ \\
$k$ & Thermal conductivity & $\mathrm{W} / \mathrm{mK}$ \\
$\Delta p$ & Pressure drop & $\mathrm{Pa}$ \\
$\mathrm{P}_{\text {pump }}$ & Pumping power & $\mathrm{W}$ \\
$q$ & Heat flux & $\mathrm{W} / \mathrm{m}^{2}$ \\
$Q$ & Power dissipation & $\mathrm{W}$ \\
$R$ & Thermal resistance & $\mathrm{K} / \mathrm{W}$ \\
$\mathrm{Re}$ & Reynold's number & - \\
$\Delta T$ & Temperature rise & $\mathrm{K}$ \\
$v$ & Velocity & $\mathrm{m} / \mathrm{s}$ \\
\hline
\end{tabular}

\section{INTRODUCTION}

Wide band-gap semiconductors, such as $\mathrm{GaN}$ and $\mathrm{SiC}$, have the potential to dramatically reduce the footprint of power devices, such as transistors and diodes, due to their larger voltage blocking capabilities [1]. Ongoing developments in lateral $\mathrm{GaN}$ power devices, such as improved epi-layers, field plates and tri-gates, are resulting in reduced specific onresistance for a given voltage rating [2], [3]. Especially with the introduction of new power transistors and diodes based on $\mathrm{GaN}$ superlattice heterostructures, recently proposed in the literature [4], [5], major improvements in the $\mathrm{V}_{\mathrm{br}} / \mathrm{R}_{\text {on }}$ figure of merit has been realized. The subsequent reduction in device size in combination with the lateral nature of GaN power HEMTs enable monolithic integration of multiple components into compact power ICs with considerably lower parasitic inductance [6], thus allowing much higher frequency operation as well as high power density. These advances open doors to a future where a complete high power converter can be integrated on a single chip [7], [8], by following the same path that logic ICs have taken over the last six decades. However, such converters require orders of magnitude higher currents compared to logic ICs. In combination with the reduced die size and the higher level of integration, this results in a significantly higher heat flux, which ultimately degrades device performance due to self-heating, limiting miniaturization and integration of these power devices.

The absence of efficient and cost-effective thermal management solutions makes massive integration of high power devices unattainable. Current successful solutions for handling extreme heat fluxes in GaN devices, relying on high thermal conductivity substrates such as $\mathrm{SiC}(380 \mathrm{~W} / \mathrm{mK})$ and Diamond (2200 W/mK) [9], are prohibitively expensive (Table I), which prevents widespread adoption of this technology. The

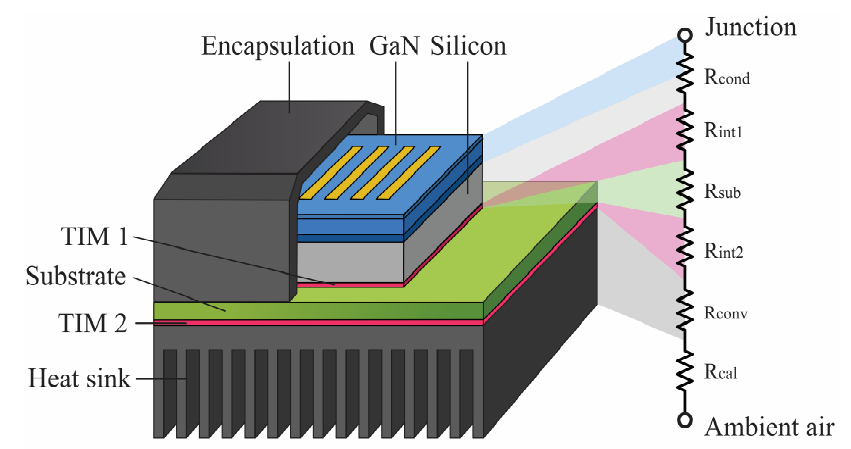

Fig. 1. Schematic illustration of an indirect cooling approach, where a chip is mounted on a PCB, and separated from a heat sink using a thermal interface material.

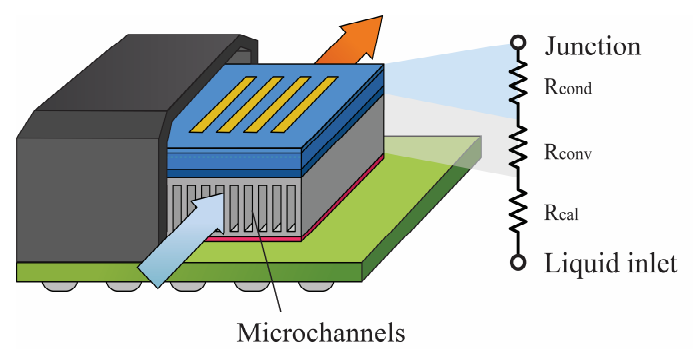

Fig. 2. Schematic illustration of a direct cooling approach, where the coolant flows in direct contact with the semiconductor die.

(C) 2020 IEEE. Personal use of this material is permitted. Permission from IEEE must be obtained for all other uses, in any current or future media, including reprinting/republishing this material for advertising or promotional purposes, creating new collective works, for resale or redistribution to servers or lists, or reuse of any copyrighted component of this work in other works." 
TABLE I

PROPERTIES OF NON-NATIVE SUBSTRATES FOR GAN ELECTRONICS

\begin{tabular}{ccc}
\hline \hline Substrate & $\boldsymbol{k}[\mathrm{W} / \mathbf{m k}]$ & Cost $\left[\mathbf{\$} / \mathbf{c m}^{\mathbf{2}}\right]$ \\
\hline $\mathrm{Si}$ & 150 & $10^{0}$ \\
$\mathrm{SiC}$ & 380 & $10^{1}$ \\
Diamond & 2200 & $10^{3}$ \\
\hline
\end{tabular}

silicon substrate has a significantly lower thermal conductivity $(150 \mathrm{~W} / \mathrm{mK})$, but is orders of magnitude more cost effective. As such, most successfully commercialized GaN devices for power electronics applications have focused on using a silicon substrate [10]. Yet, the substrate provides no functionality to the electronic device, other than carrying the few micrometers of $\mathrm{AlGaN} / \mathrm{GaN}$ epilayer that provides the active surface for the electronics.

In a conventional indirect cooling configuration (Fig. 1), the heat flux generated in the active layer propagates through multiple materials and interfaces before reaching the ambient air, resulting in a large thermal resistance. A more efficient structure has the heat sink directly embedded inside the chip, called direct cooling (Fig. 2), which removes most of the thermal resistance components. Direct cooling approached based on microchannels [11], [12], pin fins [13]-[15], impinging jets [16] and manifold microchannels [17]-[20] have been successfully demonstrated to very high heat fluxes, surpassing $1 \mathrm{~kW} / \mathrm{cm}^{2}$. The well-established, low-cost MEMS micro-fabrication processes developed for silicon can be used to turn the substrate into a high-performance liquid-cooled microchannel heat sink [21] with state-of-the art cooling capabilities.

The purpose of this work is to demonstrate a new thermal management approach for direct cooling of $\mathrm{GaN}$-on-Si power ICs, where the Si substrate functions as a microchannel heat sink (Fig. 3(a)). This approach turns GaN-on-Si into a costeffective, high thermal performance substrate, allowing a much denser integration of power devices in a single chip. As a proof of concept, we demonstrate the thermal and electrical performances of an integrated FWBR, based on high performance tri-anode GaN SBDs, with embedded microfluidic cooling, which is compared to conventional cooling methods. To showcase the feasibility of direct cooling on high powerdensity converters, this liquid-cooled power IC is integrated on a novel hybrid coolant-delivery PCB that provides both fluidic and electronic interconnection between the IC and the outside world, using easy-to-use fabrication methods.

\section{FABRICATION}

This section describes the step-by-step procedures to fabricate a GaN-on-Si power IC with substrate-embedded microchannel heat sink, as well as the procedure to assemble a multi-layer PCB with embedded coolant delivery channels which was used to interface the power IC.

\section{Device fabrication}

Tri-anode SBD FWBR were fabricated on an AlGaN/GaNon-silicon wafer, according the process previously described in [22] and summarized in Fig. 3(b). The silicon substrate was 1 mm-thick, on which a $4.2 \mu \mathrm{m}$-thick buffer layer was epitaxially grown. The GaN channel was $420 \mathrm{~nm}$ thick, followed by a 20 $\mathrm{nm}$ AlGaN barrier and a $2.9 \mathrm{~nm} \mathrm{GaN}$ cap layer. This structure results in a 2-dimensional electron gas (2-DEG), a high

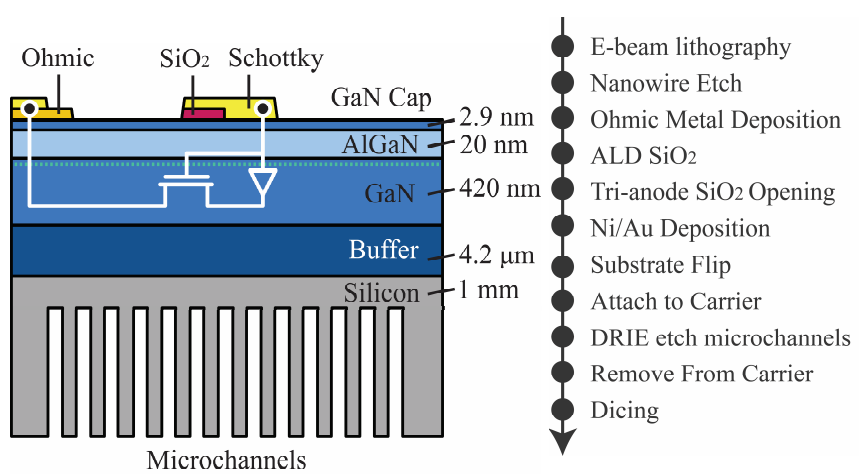

(a)

(b)

Fig. 3. (a) Schematic illustration of the liquid cooled power IC, where the silicon substrate functions as the cooling layer and the $\mathrm{AlGaN} / \mathrm{GaN}$ epilayer provides the electronic properties. (b) Overview of the fabrication steps for the liquidcooled GaN-on-Si power IC.

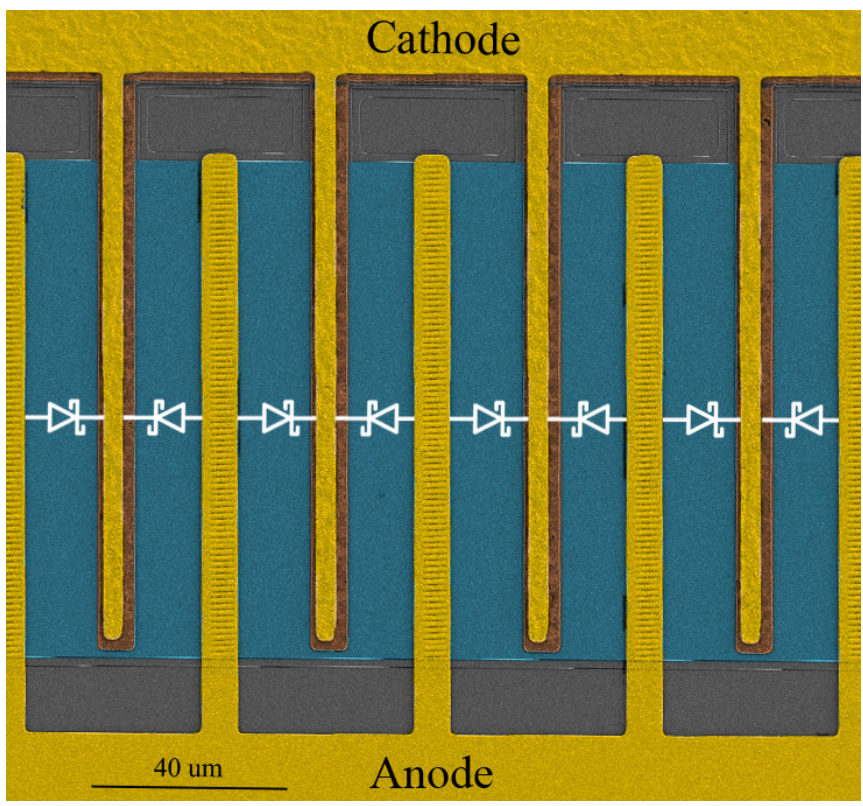

Fig. 4. SEM image of the fabricated GaN SBD. The area marked in yellow indicates the metallization of the anode and cathode region. The area marked in blue indicates the active area of the device where a 2DEG is present, and the area marked in brown indicates the annealed ohmic contact.

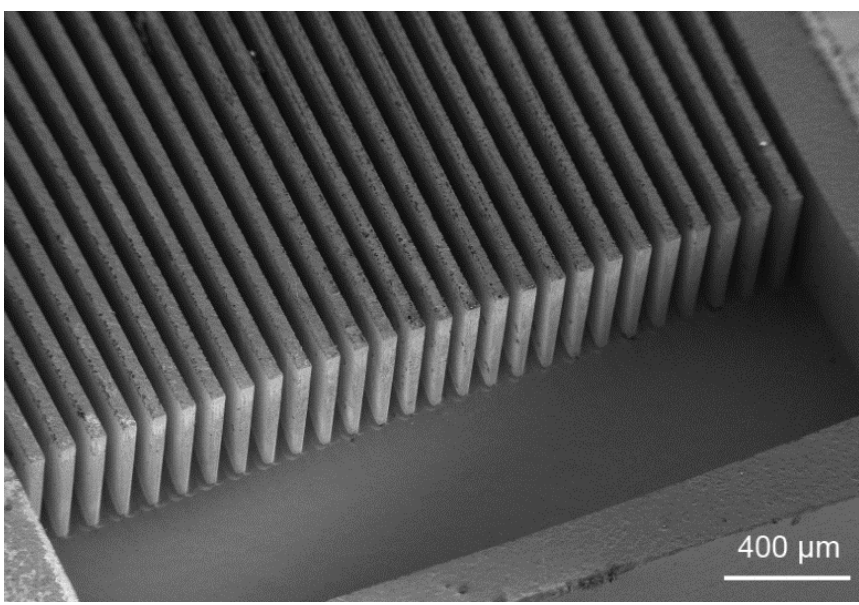

Fig. 5. High aspect ratio microchannels etched in the backside of the power IC.

(C) 2020 IEEE. Personal use of this material is permitted. Permission from IEEE must be obtained for all other uses, in any current or future media, including reprinting/republishing this material for advertising or promotional purposes, creating new collective works, for resale or redistribution to servers or lists, or reuse of any copyrighted component of this work in other works." 
concentration of electrons $\left(1.25 \cdot 10^{13} \mathrm{~cm}^{-2}\right)$ with high mobility $\left(1700 \mathrm{~cm}^{2} \mathrm{~V}^{-1} \mathrm{~s}^{-1}\right)$ at the interface between $\mathrm{AlGaN}$ and GaN. A nanowire structure was realized in the anode region of the SBD in order to obtain high breakdown performance and to reduce turn-on voltage, as previously demonstrated in [22], [23]. The nanowires were patterned using E-beam lithography, to reach the desired nanowire width and spacing of respectively $200 \mathrm{~nm}$ and $100 \mathrm{~nm}$, followed by an inductively coupled plasma (ICP) etching step. Next, a metal stack (Ti/Al/Ti/Ni/Au) was deposited onto the cathode region using e-beam physical vapor deposition (PVD), followed by an annealing step to form an ohmic contact. $\mathrm{A} \mathrm{SiO}_{2}$ dielectric layer was formed on the surface using atomic layer deposition (ALD), followed by an etching step to open the anode and cathode region. Finally, the SBD FWBR was finished by depositing a layer of $\mathrm{Ni} / \mathrm{Au}$ on the anode region to form a Schottky contact, using PVD. Fig 4 shows a SEM image of a section of a single SBD, part of the FWBR, where the tri-anode and tri-gate regions can be observed on the active area of the anode pad as subtle lines.

The epilayer was coated with a photoresist as a protective layer, before flipping the substrate and temporarily bonding it to a carrier wafer. This enables the fabrication process on the silicon substrate. Microchannel structures with a channel width and spacing of $50 \mu \mathrm{m}$ were patterned using optical lithography, followed by a deep reactive ion etching step (DRIE) to create high aspect ratio microchannels with a depth of $500 \mu \mathrm{m}$. Finally, the substrate was detached from the carrier wafer and diced into individual power ICs. Fig. 5 shows a SEM of the sharp sidewall profile of the microchannels etched in the back of the power IC, as well as the inlet cavity, which was $0.8 \mathrm{~mm}$ by $2.7 \mathrm{~mm}$.

\section{PCB fabrication}

In order to deliver the coolant to the power IC, an interface is required between the small openings of the microchannels and the larger-diameter tubes that supplies the coolant. Multiple strategies have been proposed in the literature such as Poly(methyl methacrylate) (PMMA) or polycarbonate manifolds that can interface with the device, while fluidic connectors as well as sensors can be threaded into such manifolds [24]. However, since the objective is to maximize power density, these approaches add a large volume of unused material. To improve power density, a new approach was explored where the PCB was used to provide both electric and fluidic interface to the power IC. This was achieved by fabricating 3 separate PCBs, as shown in Fig. 6. The top PCB has two cavities that are aligned with the inlet and outlet cavities of the microchannels, with dimensions of $0.7 \mathrm{~mm}$ by $2.5 \mathrm{~mm}$. This top PCB also contains the wire-bond pads to create an electrical connection with the device. Coolant inlet and outlet holes with a diameter of $9 \mathrm{~mm}$ are surrounded by an exposed soldering pad such that connectors can be soldered onto the PCB. The middle PCB provides 4 electrical input and output terminals, as well as a cutout that functions as a channel to guide the coolant to the chip. All parts of the PCB that are exposed to the coolant are gold-plated to prevent oxidation. The purpose of the bottom layer is to seal the coolant delivery channels in the middle PCB.

The three PCB are bonded together using laser-cut doublesided adhesive, as shown in Fig. 7. First, the PCBs were

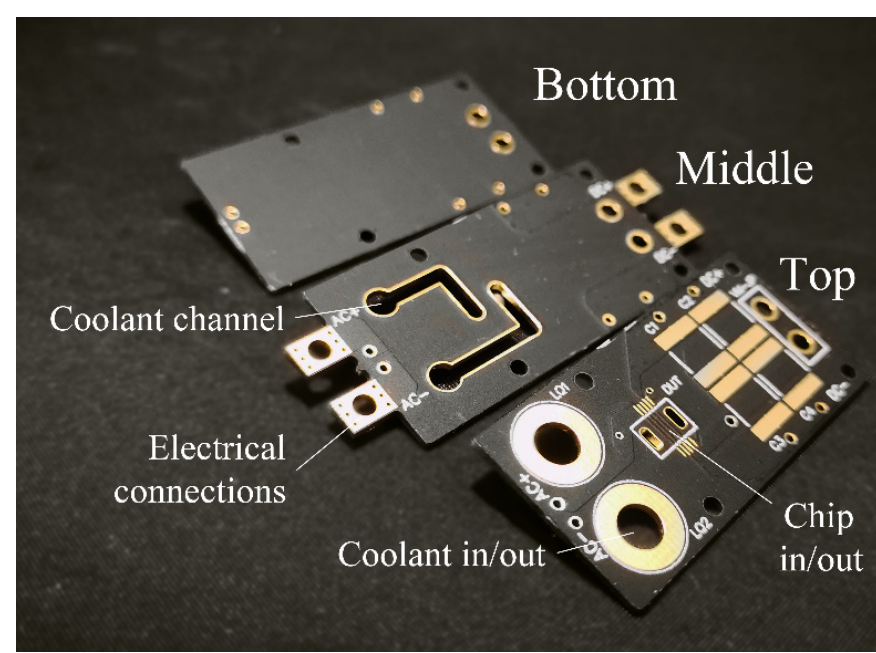

Fig. 6. 3 PCBs that together provide electrical and fluidic interconnects to the power IC

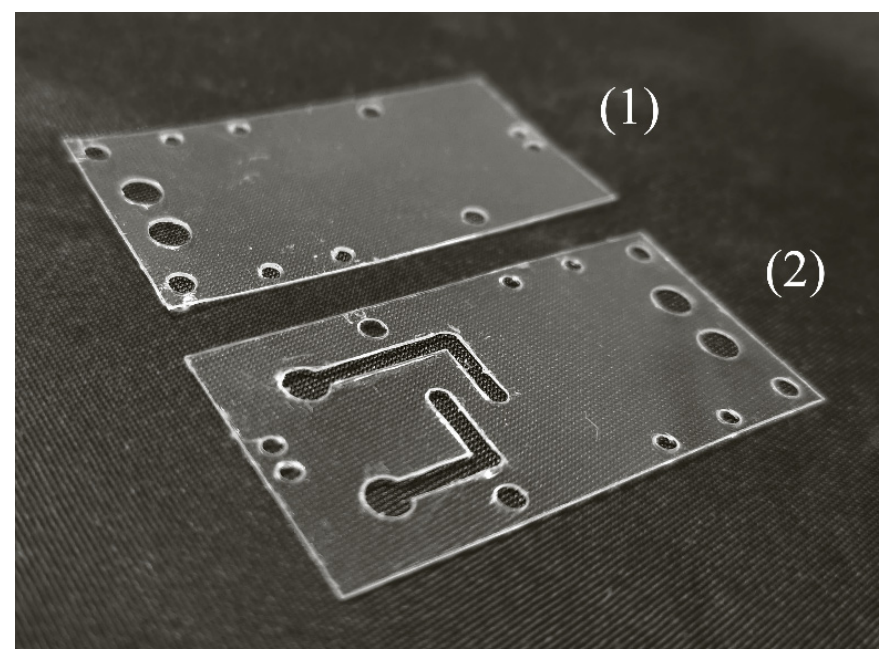

Fig. 7. Laser-cut double-sided adhesive to connect the 3 PCBs together.

thoroughly cleaned using IPA and fiber-free cloth, to ensure a good leak-tight bond. The selected adhesive (Adhesives Research ARseal 90880) can withstand temperatures up to $120^{\circ} \mathrm{C}$, rapid thermal cycling and is resistant to dilute solutions of alcohols and organic solvents. After bonding the three layers, the assembly was put in a vice for 30 minutes to ensure a proper seal.

Finally, M3 female threads were soldered onto the inlet and outlet holes to attach barbed connectors, and output capacitors were mounted on the PCB to smoothen the output signal of the power device. Finally, the device was attached to the PCB using double-sided adhesive, and wire-bonded.

\section{EXPERIMENTAL SETUP}

The assembled power IC with PCB embedded cooling, as shown in Fig. 8, was experimentally evaluated using the setup shown in Fig. 9. The aim of this setup is to investigate the cooling performance of the microchannel heat sink. A regulated DC power supply (TTI QPX1200S) was connected to the diode bridge such that all diodes are conduction. This way, an equal distribution of losses can be generated over the 4 diodes during DC operation, and the amount of dissipated power can be accurately monitored. An open loop, single phase cooling 


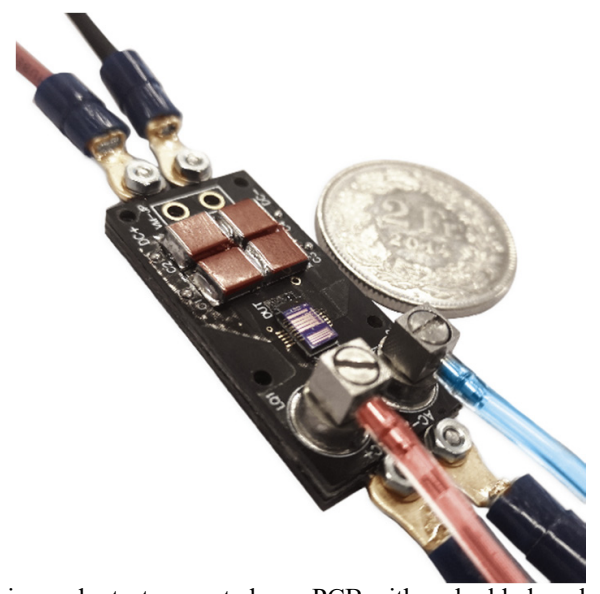

Fig. 8. Device under test, mounted on a PCB with embedded coolant delivery channels. False coloring on the inlet and outlet tubes indicate the cold coolant entering the PCB, and the hot coolant leaving the PCB.

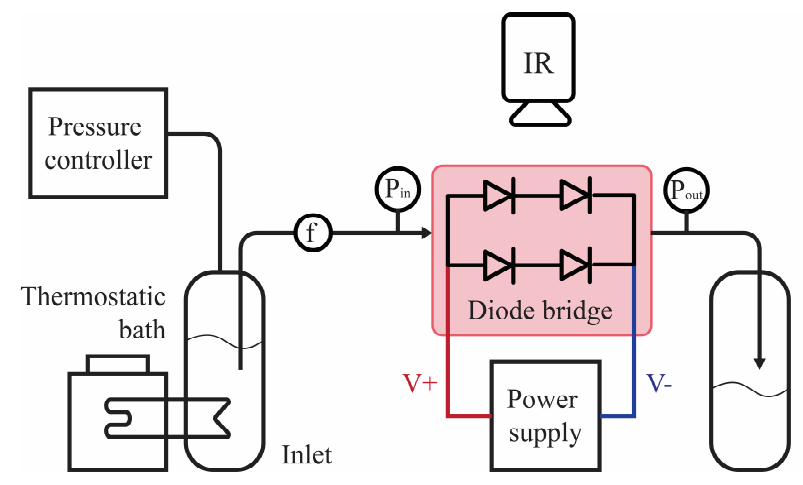

Outlet

Fig. 9. Schematic illustration of the experimental setup for evaluating the liquid cooled power IC.

system was employed, using de-ionized (DI) water as a coolant. A pressure controller (Elveflow OB1 MK3) was used to pressurize the inlet reservoir of coolant, which was kept at a constant temperature using a thermostatic bath (LAUDA Proline RP845). By pressurizing the inlet reservoir, coolant starts flowing through the PCB, after passing a flowmeter and pressure sensor at the inlet. An infrared (IR) microscope with high resolution (QFI InfraScope) was used to map the temperature profile at the surface of the chip during operation. The power IC was coated with black spray paint to increase emmisivity, and pixel-by-pixel emissivity correction was applied to ensure accurate temperature readings.

\section{RESULTS AND DISCUSSION}

The GaN-on-Si power IC with substrate-embedded microchannel heat sink was hydraulically and thermally evaluated to assess its performance. The results are discussed in this section, and compared to the literature.

TABLE II

MEASUREMENT CONDITIONS AND RESULTS

\begin{tabular}{cccccccc}
\hline \hline $\begin{array}{c}\boldsymbol{f} \\
{[\mathbf{m} \mathbf{l} / \mathbf{s}]}\end{array}$ & $\begin{array}{c}\boldsymbol{v} \\
{[\mathbf{m} / \mathbf{s}]}\end{array}$ & $\begin{array}{c}\boldsymbol{R} \boldsymbol{e} \\
{[-]}\end{array}$ & $\begin{array}{c}\boldsymbol{R}_{\text {th }} \\
{[\mathbf{K} / \mathbf{W}]}\end{array}$ & $\begin{array}{c}\Delta \boldsymbol{p} \\
{[\mathbf{m b a r}]}\end{array}$ & $\begin{array}{c}\mathbf{Q}_{\max } \\
{[\mathbf{W}]}\end{array}$ & $\begin{array}{c}\mathbf{P}_{\text {pump }} \\
{[\mathbf{m W}]}\end{array}$ & $\begin{array}{c}\mathbf{C O P}_{\mathbf{6 0}} \\
{[-]}\end{array}$ \\
\hline 0.08 & 0.11 & 10 & 4.9 & 32 & 12.2 & 0.26 & 57991 \\
0.14 & 0.20 & 18 & 3.5 & 73.2 & 17.1 & 1.0 & 19619 \\
0.30 & 0.43 & 39 & 2.8 & 181 & 21.4 & 5.4 & 4937 \\
0.52 & 0.74 & 67 & 2.6 & 360 & 23.1 & 19 & 1613 \\
0.83 & 1.19 & 108 & 2.4 & 733 & 25 & 61 & 537 \\
\hline \hline
\end{tabular}

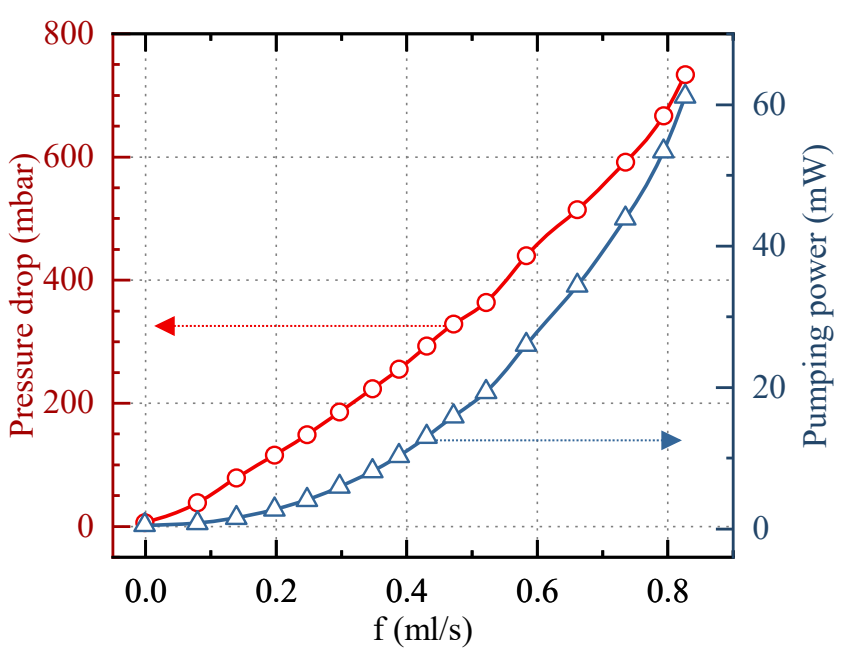

Fig. 10. Pressure drop (left) and pumping power (right) for flow rates up to $0.83 \mathrm{ml} / \mathrm{s}$.

\section{Pressure drop and pumping power}

A range of flow rates from $0.08 \mathrm{ml} / \mathrm{s}$ to $0.83 \mathrm{ml} / \mathrm{s}$ was experimentally evaluated. Given the dimensions of the microchannels of $50 \mu \mathrm{m} \times 500 \mu \mathrm{m}$, and a total of 28 parallel channels, this corresponds to an average velocity between 0.11 $\mathrm{m} / \mathrm{s}$ and $1.19 \mathrm{~m} / \mathrm{s}$ in the microchannels. These results are summarized in Table II. The hydraulic diameter of the microchannels was $91 \mu \mathrm{m}$. Therefore, this range of flow-rates corresponds to a Reynold's number from 10 to 108, well within the limits of laminar flow. The pressure drop increased linearly from $32 \mathrm{mbar}$ at $0.08 \mathrm{ml} / \mathrm{s}$ to $733 \mathrm{mbar}$ at $0.83 \mathrm{ml} / \mathrm{s}$, as shown in Fig. 10. These values corresponds to a hydraulic resistance of $8.7 \pm 33 \cdot 10^{10} \mathrm{~Pa} \cdot \mathrm{s} \cdot \mathrm{m}^{-3}$. Ideal pumping power was calculated using (1), the product of flow rate and pressure drop, and is shown in Fig. 10, as well as summarized in Table II. To achieve the maximum flow rate of $0.83 \mathrm{ml} / \mathrm{s}$, only $61 \mathrm{~mW}$ of pumping power was required, a value that is easily attainable using smallsized micropumps.

$$
P_{\text {pump }}=f \Delta p
$$

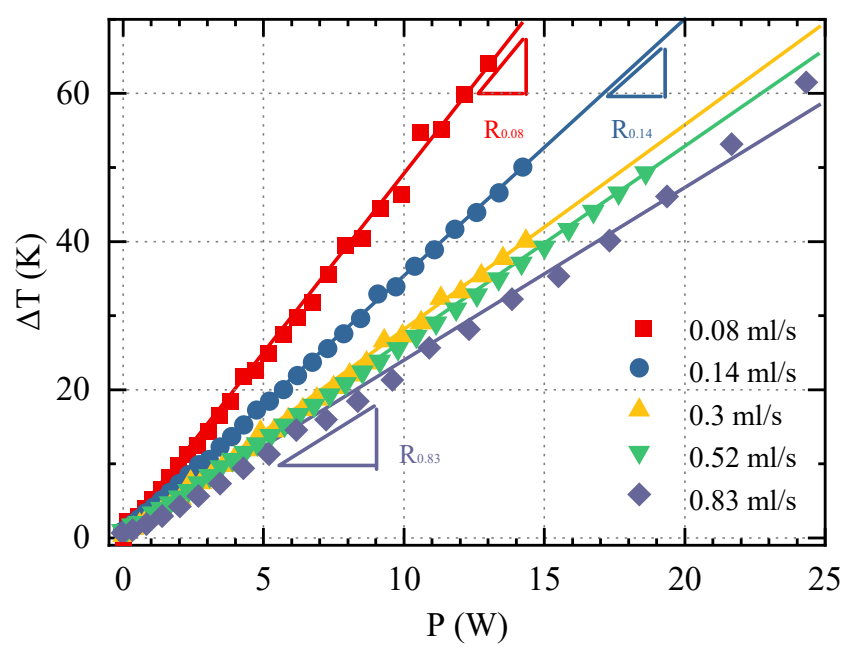

Fig. 11. Temperature rise versus power dissipation for flow rates between 0.08 and $0.83 \mathrm{ml} / \mathrm{s}$. Slopes of the linear fit through the data points indicate the thermal resistance at a given flow rate.

(C) 2020 IEEE. Personal use of this material is permitted. Permission from IEEE must be obtained for all other uses, in any current or future media, including reprinting/republishing this material for advertising or promotional purposes, creating new collective works, for resale or redistribution to servers or lists, or reuse of any copyrighted component of this work in other works." 


\section{Temperature rise and thermal resistance}

For each condition of flow-rate, the DC power dissipation on the power IC was gradually increased until the surface temperature rise, monitored using the calibrated IR camera, reached a value of $60 \mathrm{~K}$. Surface temperature rise versus power dissipation for varying flow rates is shown in Fig. 11. At a flow rate of $0.83 \mathrm{ml} / \mathrm{s}$, up to $25 \mathrm{~W}$ of losses were extracted from the $2.7 \times 3.0 \mathrm{~mm}^{2} \mathrm{IC}$, corresponding to a device-level heat flux of $417 \mathrm{~W} / \mathrm{cm}^{2}$, far beyond the capabilities of conventional indirect cooling methods. The temperature rise shows a linear relation versus power dissipation, and the slope of the linear fit through these data points yields the thermal resistance at a specific flow rate condition.

The obtained thermal resistance versus flow rate is plotted in Fig. 12, demonstrating a clear linear relationship, defined by the caloric thermal resistance $\left(R_{c a l}\right)$ of the coolant, which here was de-ionized water. The intercept with the y-axis indicates the thermal resistance at infinite flow rate, when $R_{\text {cal }}$ approaches zero, which is approximately $2 \mathrm{~K} / \mathrm{W}$. This remaining part of the thermal resistance can be accounted to the convective heat transfer between the microchannel wall and the coolant $\left(R_{\text {conv }}\right)$ and to the conductive thermal resistance between the junction and the microchannels $\left(R_{\text {cond }}\right)$. Using $(2)$, an expression for the conductive thermal resistance, under the assumption of $1 \mathrm{D}$ heat transfer over a distance of $750 \mu \mathrm{m}$, shows that the contribution of $\mathrm{R}_{\text {cond }}$ is approximately $0.62 \mathrm{~K} / \mathrm{W}$. As a result, the remaining $1.4 \mathrm{~K} / \mathrm{W}$ can be accounted to the convective thermal resistance, as defined in (3).

$$
\begin{aligned}
& R_{\text {cond }}=\frac{t}{k A} \\
& R_{\text {conv }}=\frac{1}{h A}
\end{aligned}
$$

Using the footprint of the microchannels $\left(2.7 \times 3.0 \mathrm{~mm}^{2}\right)$, this leads to an effective heat transfer coefficient of $h_{\text {eff }}=8.8 \cdot 10^{4}$ $\mathrm{WK}^{-1} \mathrm{~m}^{-2}$. If the complete surface area of the walls of the microchannels is considered, this leads to an average wall heat transfer coefficient of $h_{\text {wall }}=8.0 \cdot 10^{3} \mathrm{WK}^{-1} \mathrm{~m}^{-2}$. Although this value is lower than the local heat transfer coefficient expected in typical laminar flow microchannel cooling applications, due to the fact that the limited fin efficiency lowers the effective heat transfer coefficient in high aspect ratio microchannels, it is significantly higher than any heat transfer coefficient that could be obtained using air-cooling or millimeter-sized liquid cooling solutions.

\section{Cooling coefficient of performance}

The low pumping power in combination with a low thermal resistance leads to high performance cooling of high heat fluxes. The coefficient of performance (COP), given in (4), gives the ratio of heat load that can be applied before reaching a critical temperature rise ( $60 \mathrm{~K}$ in this case), to the amount of pumping power required to achieve this level of cooling. Fig. 13 shows the COP versus heat flux. As can be seen, COP decreases rapidly with increasing heat flux. This is due to the fact that $P_{\text {pump }}$ scales with $f^{2}$, whereas Q $\mathrm{Qmax}_{\text {on }}$ on scales with $f^{1}$. It is therefore required to assess COP at multiple measurement conditions, as efficiency depends on the given flow-rate.

$$
C O P_{60}=\frac{Q_{\max }}{P_{\text {pump }}}=\frac{60}{f \Delta p R_{t}}
$$

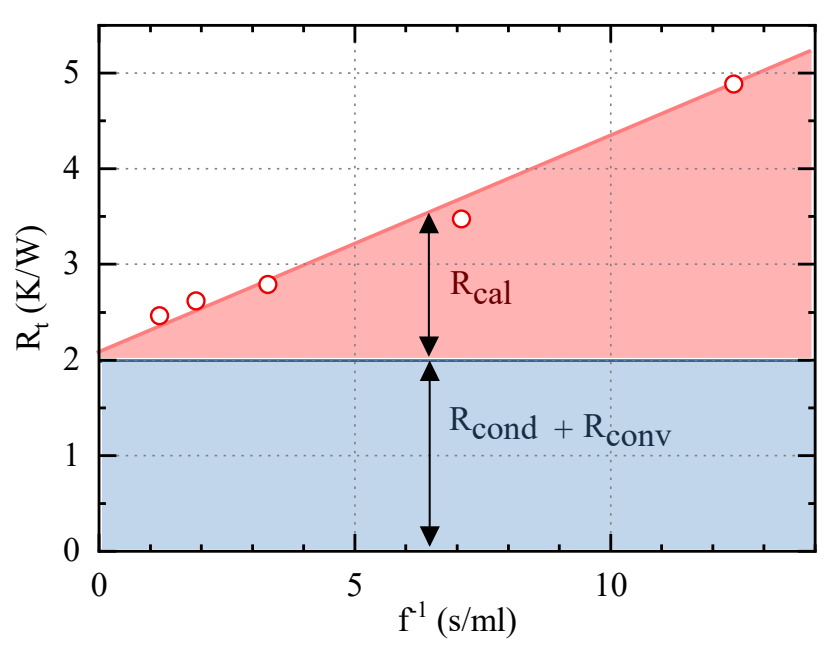

Fig. 12. Thermal resistance versus inverse flow rate. The area marked in red indicates the contribution of the heating of the coolant $\left(R_{\text {cal }}\right)$ on the total thermal resistance, which scales with $f^{l}$. The area marked in blue indicates the remaining contribution to the thermal resistance due to conductive and convective heat transfer between the junction and the coolant.

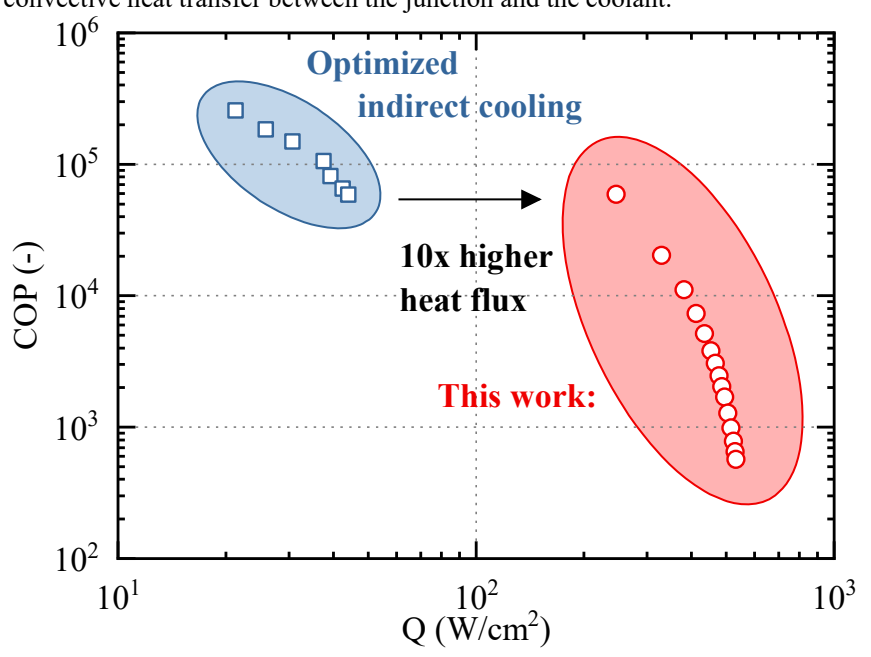

Fig. 13. Coefficient of performance versus maximum heat flux, for a device temperature rise of $60 \mathrm{~K}$. Round markers indicate the results presented in this paper, whereas square markers refer to optimized indirect microchannel cooling of $\mathrm{GaN}$ power devices, as presented in [25].

Fig. 13 also shows data points from a work in the literature that utilized similar $50 \mu \mathrm{m}$-wide silicon microchannel coldplates in an indirect cooling configuration, separated from a $\mathrm{GaN}$ transistor using a thermal interface material [25]. The direct cooling approach allows approximately 10x higher heat fluxes, due to the absence of interface thermal resistance and the typical junction-to-case thermal resistance in a packaged device. This clearly highlights the benefits in power density that can be achieved by using the silicon substrate as a microchannel heat sink.

\section{High power rectification}

To demonstrate the improved power conversion capabilities of the liquid cooled power IC, an AC waveform up to $125 \mathrm{~V}$ was fed into the FWBR at $10 \mathrm{kHz}$ and rectified into a DC output voltage. The temperature rise of the liquid cooled converter was compared to the same setup without the presence of liquid cooling, thus depending solely on natural convection. Fig. 14(a) 


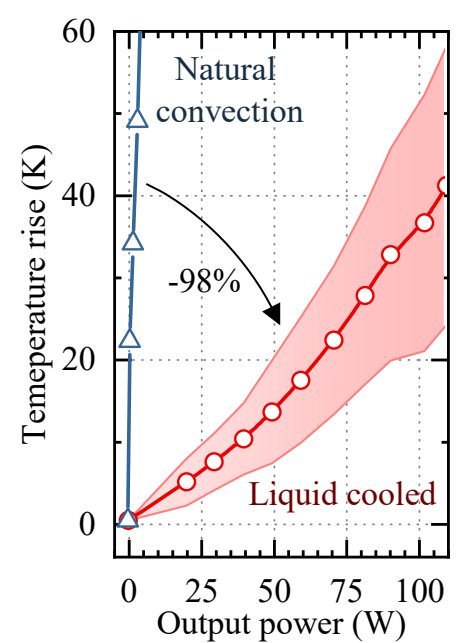

(a)

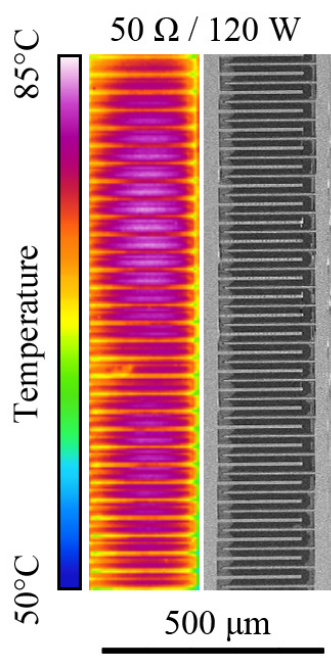

(b)
Fig. 14. (a) Temperature rise versus output power of the rectifier with substrateembedded cooling, indicated in red, and an identical device depending solely on natural convection cooling (blue). (b) IR thermograph of the surface of a single diode during $120 \mathrm{~W}$ rectification (left), SEM image of the same diode, showing the anode and cathode finger structure (right)

shows the temperature rise of the surface of the chip versus output power of the rectifier. As can be seen, up to $120 \mathrm{~W}$ of DC output power could be delivered from the FWBR with substrate-embedded microchannel cooling before reaching a peak temperature of $60 \mathrm{~K}$.

The spread in Fig. 14(a) represents the range of temperatures measured over the entire diode, as shown in Fig. 14(b). Fig 14(b) shows an SEM image of a single diode, and the corresponding IR temperature map. The markers thus represent the mean value, whereas the outer lines represent the minimum and maximum observed temperatures. The FWBR cooled by natural convection shows a significant higher temperature rise: Only $4 \mathrm{~W}$ of DC output power could be delivered before reaching the same temperature rise of $60 \mathrm{~K}$. By employing substrate-embedded microchannel cooling, a reduction of $98 \%$ in thermal resistance was observed, a promising result for the future of highly miniaturized integrated power converters.

\section{SUMMARY \& CONCLUSIONS}

In this work, the concept of substrate-embedded microchannel cooling of GaN-on-Si power ICs was demonstrated for the first time. Total device-level heat fluxes of up to $417 \mathrm{~W} / \mathrm{cm}^{2}$ were obtained, with a temperature rise 60 $\mathrm{K}$. These results show that an order of magnitude higher heat fluxes can be achieved by embedding the cooling directly into the semiconductor die. Furthermore, a new approach of utilizing the $\mathrm{PCB}$ as coolant delivery platform was presented as a very compact and low-cost approach to prototype directcooled devices. This embedded cooling approach, in combination with the realized $\mathrm{GaN}$ power IC, resulted in a 30 times increase of output power. These results demonstrate the potential to realize high-power (kW-range) converters of the size of a USB-stick in the foreseeable future.

\section{Acknowledgements}

This work was supported in part by the European Research Council under the European Union's H2020 program/ERC
Grant Agreement No. 679425, in part by the Swiss Office of Energy Grant No. SI501568-01 and in part by the Swiss National Science Foundation under Assistant Professor (AP) Energy Grant PYAPP2_166901

\section{References}

[1] H. Amano et al., "The $2018 \mathrm{GaN}$ power electronics roadmap," J. Phys. D. Appl. Phys., vol. 51, no. 16, p. 163001, Apr. 2018.

[2] S. Karmalkar and U. K. Mishra, "Enhancement of breakdown voltage in $\mathrm{AlGaN} / \mathrm{GaN}$ high electron mobility transistors using a field plate," IEEE Trans. Electron Devices, vol. 48, no. 8, pp. 1515-1521, 2001.

[3] K. J. Chen and C. Zhou, "Enhancement-mode AlGaN/GaN HEMT and MIS-HEMT technology," Phys. status solidi, vol. 208, no. 2, pp. 434-438, Feb. 2011.

[4] J. Ma, G. Kampitsis, P. Xiang, K. Cheng, and E. Matioli, "Multi-Channel Tri-Gate GaN Power Schottky Diodes With Low ON-Resistance," IEEE Electron Device Lett., vol. 40, no. 2, pp. 275-278, Feb. 2019.

[5] J. Ma, C. Erine, P. Xiang, K. Cheng, and E. Matioli, "Multi-channel tri-gate normally-on/off AlGaN/GaN MOSHEMTs on Si substrate with high breakdown voltage and low ON-resistance," Appl. Phys. Lett., vol. 113 , no. 24 , p. 242102 , Dec. 2018.

[6] N. Fichtenbaum, M. Giandalia, S. Sharma, and J. Zhang, "Half-Bridge GaN Power ICs: Performance and Application," IEEE Power Electron. Mag., vol. 4, no. 3, pp. 33-40, Sep. 2017.

[7] R. Reiner et al., "Monolithically integrated power circuits in high-voltage GaN-on-Si heterojunction technology," IET Power Electron., vol. 11, no. 4, pp. 681-688, Apr. 2018.

[8] X. Li et al., "200V enhancement-mode p-GaN HEMTs fabricated on $200 \mathrm{~mm}$ GaN-on-SOI with trench isolation for monolithic integration," IEEE Electron Device Lett., vol. 38, no. 7, pp. 918-921, Jul. 2017.

[9] M. Kuball et al., "Novel Thermal Management of GaN Electronics: Diamond Substrates," in Volume 3: Advanced Fabrication and Manufacturing; Emerging Technology Frontiers; Energy, Health and WaterApplications of Nano-, Micro- and Mini-Scale Devices; MEMS and NEMS; Technology Update Talks; Thermal Management Using Micro Channels, Jets, Sprays, 2015, p. V003T08A001.

[10] K. J. Chen et al., "GaN-on-Si Power Technology: Devices and Applications," IEEE Trans. Electron Devices, vol. 64, no. 3, pp. 779-795, Mar. 2017.

[11] D. B. Tuckerman and R. F. W. Pease, "Highperformance heat sinking for VLSI," IEEE Electron Device Lett., vol. 2, no. 5, pp. 126-129, May 1981.

(C) 2020 IEEE. Personal use of this material is permitted. Permission from IEEE must be obtained for all other uses, in any current or future media, including reprinting/republishing this material for advertising or promotional purposes, creating new collective works, for resale or redistribution to servers or lists, or reuse of any copyrighted component of this work in other works." 
[12] R. J. Phillips, "Michrochannel Heat Sinks," Lincoln Lab. J. (ISSN 0896-4130), vol. 1, Spring 1988, p. 31 48., vol. 1, pp. 31-48, 1988.

[13] Y. Zhang, A. Dembla, and M. S. Bakir, "Silicon micropin-fin heat sink with integrated TSVs for 3-D ICs: Tradeoff analysis and experimental testing," IEEE Trans. Components, Packag. Manuf. Technol., vol. 3, no. 11, pp. 1842-1850, 2013.

[14] D. Kong et al., "Single-phase thermal and hydraulic performance of embedded silicon micro-pin fin heat sinks using R245fa," Int. J. Heat Mass Transf., vol. 141, pp. 145-155, Oct. 2019.

[15] C. Falsetti, M. Magnini, and J. R. Thome, "Hydrodynamic and thermal analysis of a micro-pin fin evaporator for on-chip two-phase cooling of high density power micro-electronics," Appl. Therm. Eng., vol. 130, pp. 1425-1439, Feb. 2018.

[16] A. J. Robinson, W. Tan, R. Kempers, J. Colenbrander, N. Bushnell, and R. Chen, "A new hybrid heat sink with impinging micro-jet arrays and microchannels fabricated using high volume additive manufacturing," in 2017 33rd Thermal Measurement, Modeling \& Management Symposium (SEMI-THERM), 2017, pp. 179-186.

[17] G. M. Harpole and J. E. Eninger, "Micro-channel heat exchanger optimization," in 1991 Proceedings, Seventh IEEE Semiconductor Thermal Measurement and Management Symposium, 2002, pp. 59-63.

[18] M. Ohadi, K. Choo, S. Dessiatoun, and E. Cetegen, "Next Generation Microchannel Heat Exchangers," in SpringerBriefs in Applied Sciences and Technology, no. 9781461407782, New York, NY: Springer New York, 2013, pp. 1-111.

[19] W. Escher, T. Brunschwiler, B. Michel, and D. Poulikakos, "Experimental Investigation of an Ultrathin Manifold Microchannel Heat Sink for Liquid-Cooled Chips," J. Heat Transfer, vol. 132, no. 8, p. 081402, Aug. 2010.

[20] L. Everhart, N. Jankowski, B. Geil, A. Bayba, D. Ibitayo, and P. McCluskey, "Manifold Microchannel Cooler for Direct Backside Liquid Cooling of SiC Power Devices," in ASME 5th International Conference on Nanochannels, Microchannels, and Minichannels, 2007, pp. 285-292.

[21] R. A. Lawes, "Manufacturing costs for microsystems/MEMS using high aspect ratio microfabrication techniques," Microsyst. Technol., vol. 13, no. 1, pp. 85-95, 2007.

[22] L. Nela, G. Kampitsis, J. Ma, and E. Matioli, "Fastswitching Tri-Anode Schottky Barrier Diodes for monolithically integrated GaN-on-Si power circuits," IEEE Electron Device Lett., pp. 1-1, 2019.

[23] J. Ma and E. Matioli, "High-voltage and low-leakage
AlGaN/GaN tri-anode schottky diodes with integrated tri-gate transistors," IEEE Electron Device Lett., vol. 38, no. 1, pp. 83-86, Jan. 2017.

[24] A. Sridhar, A. Viricenzi, M. Ruggiero, T. Brunschwiler, and D. Atienza, "3D-ICE: Fast compact transient thermal modeling for 3D ICs with inter-tier liquid cooling," in IEEE/ACM International Conference on Computer-Aided Design, Digest of Technical Papers, ICCAD, 2010, pp. 463-470.

[25] R. van Erp, G. Kampitsis, and E. Matioli, “A manifold microchannel heat sink for ultra-high power density liquid-cooled converters," in 2019 IEEE Applied Power Electronics Conference and Exposition (APEC), 2019, pp. 1383-1389.

(C) 2020 IEEE. Personal use of this material is permitted. Permission from IEEE must be obtained for all other uses, in any current or future media, including reprinting/republishing this material for advertising or promotional purposes, creating new collective works, for resale or redistribution to servers or lists, or reuse of any copyrighted component of this work in other works." 